\title{
NOTICIA SOBRE UNO DE LOS LIBROS DE LA VISITA PASTORAL HECHA AL ARZOBISPADO ZARAGOZANO LOS AÑOS 1731 A 1734
}

\author{
Pilar Pueyo Colomina \\ Universidad de Zaragoza
}

Entre los fondos del Archivo Diocesano de Zaragoza se conserva, en la sección de Visitas Pastorales, un manuscrito que no tiene signatura; está compuesto por nueve cuadernillos, de los que la foliación de los ocho primeroes actual y el último está sin foliar. Su distribución es: 2 $\mathrm{XVI}\left(32^{\prime}-64^{\prime}\right)+\left(1 \mathrm{XIV}\left(92^{\prime}\right)+1 \mathrm{XI}\left(114^{\prime}\right)+1 \mathrm{XII}\left(138^{\prime}\right)+2 \mathrm{XVI}\left(170^{\prime}-202^{\prime}\right)+1\right.$ XVI-2(232') +1 XIII (s.f.).

En el cuarto cuadernillo quedan restos como si hubiera sido arrancado un folio de cada mitad de éste (entre los folios 98-99 y 109-110). El octavo era de 16 hojas, pero las que forman los folios 203 y 204 no continúan en su segunda mitad; queda el talón. El último cuadernillo era de 13 hojas, no se conserva completo, sólo restos que nos permiten conocer su composición: de la primera mitad, quedan nueve folios más fragmentos de los cuatro restantes que la componen; y de la segunda diez folios completos y parte de tres.

Los cuadernillos están cosidos con hilo de cáñamo en tres nervaturas que miden, en total, $120 \mathrm{~mm}$ : la primera y tercera, $30 \mathrm{~mm}$. y la central $60 \mathrm{~mm}$., distando las suturas inferior y superior de sus respectivos bordes $40 \mathrm{~mm}$. en cada lado. Están agrupados bajo cubiertas de pergamino de color natural, pero con abundantes manchas.

En la cubierta anterior hay un asterisco, una suma en la parte superior y cifras sueltas más o menos borrosas. En la posterior, dos cifras en el margen superior, claras, y sumas en dirección transversal en tinta muy desvaída.

Está reforzado el lomo con dos trozos rectangulares de piel cosidos con hilo de cáñamo. Estos trozos miden $35 \mathrm{~mm}$. de largo y la anchura no 
se puede dar con precisión porque forma una concavidad. Están separados entre ellos $55 \mathrm{~mm}$. y distan de los bordes superior e inferior $40 \mathrm{~mm}$.

En el lomo está escrito en dirección longitudinal, en tinta negra y posterior al manuscrito: «Visita limosnas 1731-34».

La encuadernación es en forma de cartera y las medidas de la cubierta son de $204 \times 130 \mathrm{~mm}$. la anterior, y la posterior $204 \times 214 \mathrm{~mm}$. Quedan restos de cierres; aproximadamente a mitad de la cubierta hay un agujero circular de cuatro mm. y a esta misma altura, en el interior del pergamino doblado, hay un refuerzo de $10 \times 15 \mathrm{~mm}$. con el que coincide.

Todo el manuscrito está formado por hojas de papel verjurado que aparecen plegadas en tamaño octavo. El papel, de color blando aunque un tanto amarillento por la acción del tiempo, tiene puntizones a razón de trece en centímetro y corondeles separados entre sí $20 \mathrm{~mm}$.

Todos los cuadernillos tienen la misma marca de agua. Esta filigrana está colocada en la mitad del folio y su posición varía dentro del cuadernilio.
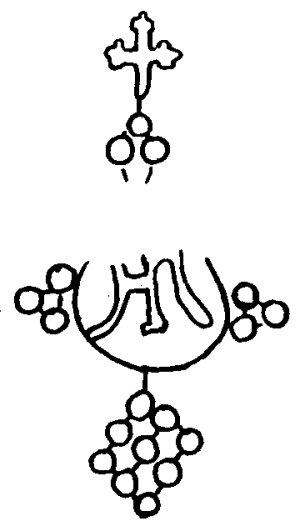

Tenemos, pues, un manuscrito, en letra humanística cursiva que ha sido ejecutado por varias manos. La cubierta no lleva título y en el lomo se lee “Visita limosnas 1731-34». En su portada interior los datos que aparecen son: «Trasobares. Antonio Urbano, molinero deve». «Urria de Jalon, molinero deve" y el resto del folio en blanco. Poco aclara sobre el contenido del mismo.

El siguiente folio está encabezado por el nombre de una parroquia de la diócesis de Zaragoza, en caracteres de mayor módulo, y tras un espacio en blanco comienza el texto. Cada línea está ocupada por el nombre y apellido de una persona seguido de una abreviatura y el nombre y apellido de otra; a continuación, una o dos cantidades separadas entre sí por 
puntos y éstas llevan sendas abreviaturas que equivalen a libras y sueldos, respectivamente. En el margen izquierdo precediendo al nombre hay una cruz y/o la letra $Y$ o una palabra abreviada. Al final figura la palabra Pila, una cantidad de dinero en sueldos y quién la debía pagar. Esto nos proporciona un dato cierto para identificar cuál es el contenido de este manuscrito.

Según a la altura del folio que se termina lo concerniente a una parroquia, se deja en blanco o se empieza con otra, dejando separación entre ambas.

La presentación del texto no es cuidada. Hay correcciones: escriben sobre lo ya escrito para rectificar los apellidos o las cifras confundidas o trazan una raya encima generalmente cuando inutilizan una línea completa. También hay anotaciones interlineadas, normalmente hechas por otra mano y son aclaraciones al texto...

Los ocho primeros cuadernillos tienen la misma estructura interna con pequeñas variantes más o menos interesantes. Por ejemplo, el nombre de la parroquia puede estar subrayad o todo, parte o nada. Pero mucho más importante es el hecho de que a partir del folio 69 al nombre de la parroquia acompañe la fecha y que aparezca ésta completa con indicación del día, mes y año en cuatro folios: $69,135,142$ y 220; en los demás sólo constan los dos primeros datos.

La aparición de la data en cuatro momentos concretos no es puro azar. El folio 69 destinado a la parroquia de Torrecilla de Valmadrid está fechado el 30 de septiembre de 1732 y en los siguientes hay nombres de otras parroquias con dias y meses correlativos hasta llegar al 135. En éste vuelve a mencionarse la fecha completa porque comienza un nuevo año. Y así consta: Caspe, 1 de enero de 1733. Después continúa hasta el 142 con más nombres de parroquias y dias sucesivos.

En este folio, la parroquia de La Muela va seguida de la fecha 21 de septiembre de 1733. Cotejando esta data con la de la parroquia inmediatamente anterior, El Burgo 11 de enero, hay un lapsus de varios meses, perfectamente explicable como ya veremos. Siguen más nombres de parroquias con dia y mes hasta llegar al folio 220 , en que vuelve a aparecer la data completa por la misma causa que en Caspe, pero ahora es Cortes de Aragón la que marca el comienzo del año 1734. Tras este folio, hasta el final del cuadernillo, continúan nombres de parroquias con día y mes.

Si todos estos nombres de parroquias, en el mismo orden del manuscrito, los plasmamos gráficamente en un mapa nos aparecerá trazado claramente un itinerario con tres rutas bien definidas (1).

La primera se inicia en Cuarte en fecha no determinada, posibleménte en el mes de septiembre (2), y la última etapa es María. La segunda ruta empieza en Torrecilla de Valmadrid el 30 de septiembre de 1732 y por ello figura la data completa, no volviéndose a indicar el año hasta Caspe. Habían transcurrido los meses de octubre, noviembre y diciembre; al 
llegar a esta localidad era el 1 de enero de 1733. Termina el periplo en El Burgo el 11 de enero. La tercera comienza en La Muela el 21 de septiembre de 1733, arriba a Cortes de Aragón el 1 de enero de 1734 y concluye en Mediana el 18 de enero de ese mismo año.

Estas tres rutas corresponden, sin duda alguna, a otras tantas salidas de una visita pastoral general a toda la diócesis realizada en los años citados. Es común que la visita general al Arzobispado de Zaragoza no se efectuara de una sola vez, sino que se dividía en varias salidas, en cada una de ellas se seguía un itinerario trazado de antemano que difería de unas a otras. Estas salidas solian hacerse en primavera y/o en otoño (3). Las de esta visita pastoral se hicieron en otoño. Esto explica la interrupción entre los meses de enero y septiembre mencionada anteriormente.

Pero ¿quién fue el autor de esta visita pastoral? Cronológicamente esta visita debe encuadrarse durante el Arzobispado de don Tomás Crespo de Agüero, el cual dirigió la diócesis de Zaragoza del año 1727 a 1742. En estos quince años se llevaron a cabo varias visitas pastorales, la primera por el propio arzobispo y las restantes por visitadores delegados de las que no queda el libro registro de la visita de ninguna de ellas en el Archivo Diocesano de Zaragoza (4).

En el manuscrito estudiado no hay ninguna referencia a su autor, pero se ha podido identificar por medio de otras fuentes, como son los mandatos de visita, documentos emanados después de esta visita y que se conservan en los archivos parroquiales. Se sabe que el visitador fue don Gregorio Galindo y, así, se intitulaba en ellos «por la gracia de Dios y de la Sede Apostólica Obispo de Aulona, Auxiliar y Visitador General del Arzobispado de Zaragoza por el IImo. Sr. D. Tomás Crespo de Agüero, Arzobispo de la misma ciudad, del Consejo de su Magestad...» (5).

Visitó 342 iglesias parroquiales. Empezó el periplo por el Arciprestazgo de Daroca, lo recorrió completo, a éste se suman quince parroquias del Arciprestazgo de Zaragoza (6); en total, 103 (7), pero se ignora el tiempo que invirtió en ello. En la segunda salida, 108 que pertenecían al Arciprestazgo de Alcañiz, aunque no lo llegó a visitar todo; las que faltaban las dejó para la siguiente (8). El itinerario duró casi tres meses y medio. Descansó del 25 de diciembre al 1 de enero en Alcañiz y en Caspe desde ese día hasta el 6.

La última salida de esta visita general la dedicó a 129 parroquias durante unos cuatro meses, casi todas eran del Arciprestazgo de Zaragoza y las que le quedaban del de Alcañiz.

El contenido de este manuscrito está directamente relacionado con esta visita pastoral de don Gregorio Galindo. Es obvio que no se trata del libro registro. Nos muestra otra faceta de la visita: su aspecto fiscal. Efectivamente, el realizar una visita general suponía grandes gastos, parte de los cuales eran subsanados con los derechos que recibia el visitador por diversos conceptos: derecho de pila, de dieta, de ducado, tres por ciento, sello de libros, de juez, de «definimientos». 
El cobro de estos derechos se apuntaba en dos libros paralelos al de la visita; uno contenía lo percibido en cada parroquia por derecho de dieta, sello de libros, de juez y «definimientos", y el otro registraba lo recibido por derecho de ducado, tres por ciento y pila (9). Por lo que se deduce que nuestro manuscrito se encuadra en esta última fuente documental.

La primera cantidad tras el nombre de la persona que lo paga se refiere al derecho de tres por ciento. Por la visita de los testamentos y de los intestados tenia la Dignidad el derecho de tres por ciento de todo lo que importaba lo pío que pagaban los herederos o fianzas que estaban puestos en los Cinco Libros, más cuatro dineros al fiscal de cada testamento o intestato (10). Pero si el fallecido era clérigo, además del tres por ciento, debía pagar por legitima el breviario y bonete, o dar por el breviario dos reales de a ocho y por el bonete cinco sueldos (11). En esta visita pastoral hemos contabilizado 136 legítimas que se pagaron en 91 de las parroquias visitadas.

La segunda, al derecho de ducado. La sepultura común de los fieles era el cementerio, pero si éstos preferían enterrarse en su iglesia parroquial debian pagar por ello. Las constituciones sinodales de este Arzobispado especificaban que «el derecho de fracción de sepultura de los párvulos y de los adultos que estuvieren debaxo la tutela de sus padres, y sin aver tomado estado, y que se enterrasen en la iglesia sea solamente onze sueldos; y assi mismo mandamos que el aniversario de los dichos adultos sea rezado entregando la parte correspondiente a quatro sueldos de renta en cada un año, y el derecho de jocalia veinte y cinco sueldos" (12).

Al final de cada parroquia consta lo que debe pagar por el derecho de pila. Las sinodales no señalaban la cantidad que correspondía a cada una y en cuanto a quién pertenecía hacerlo, mandaban que se observaran las costumbres locales (13).

Pero lo más interesante de este manuscrito no es tanto el conocimiento de los ingresos que percibió el visitador por el cobro de estos derechos, como el poder reconstruir el itinerario que don Gregorio Galindo siguió en este cometido espiritual, aspecto éste que suele proporcionarnos el libro registro de actas de visita, fuente paralela a la aquí estudiada, pero que, como hemos dicho, no se conserva por lo que respecta al caso concreto de la realizada durante los años 1731 a 1734.

ITINERARIO DE LA VISITA REALIZADA DE 1731 A 1734

$\begin{array}{ll}\text { Año Mes Día } & \text { Parroquia } \\ & \text { Cuarte } \\ & \text { Cadrete } \\ & \text { Jaulín } \\ & \text { Villanueva de Huerva }\end{array}$




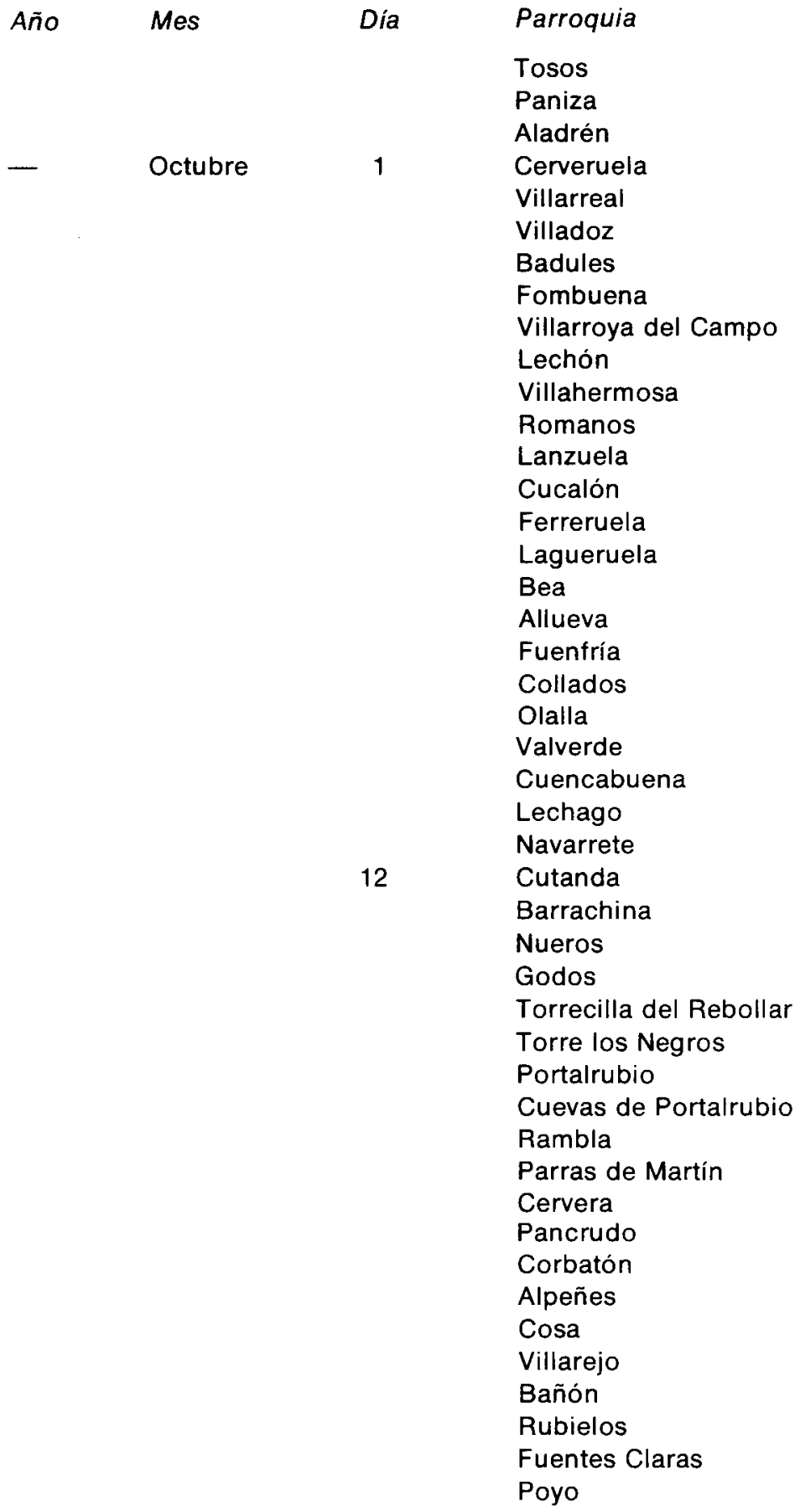


Caminreal

Torrijo

Monreal del Campo

Villafranca del Campo

Singra

Almohaja

Peracense

Ojos Negros

Villar del Salz

Pozuel

Blancas

Torralba de los Sisones

Villalba

Bello

Cuerlas

Odón

Torralba de los Frailes

Used

Santed

Gallocanta

Berrueco

Tornos

Castejón de Tornos

Calamocha

Luco

Burbáguena

Báguena

Anento

S. Martín del Río

Val de San Martín

Valdehorna

Villanueva de Jiloca

Daroca

Balconchán

Orcajo

Nombrevilla

Retascón

Manchones

Murero

Villafeliche

Langa

Torralbilla

Mainar

Codos 


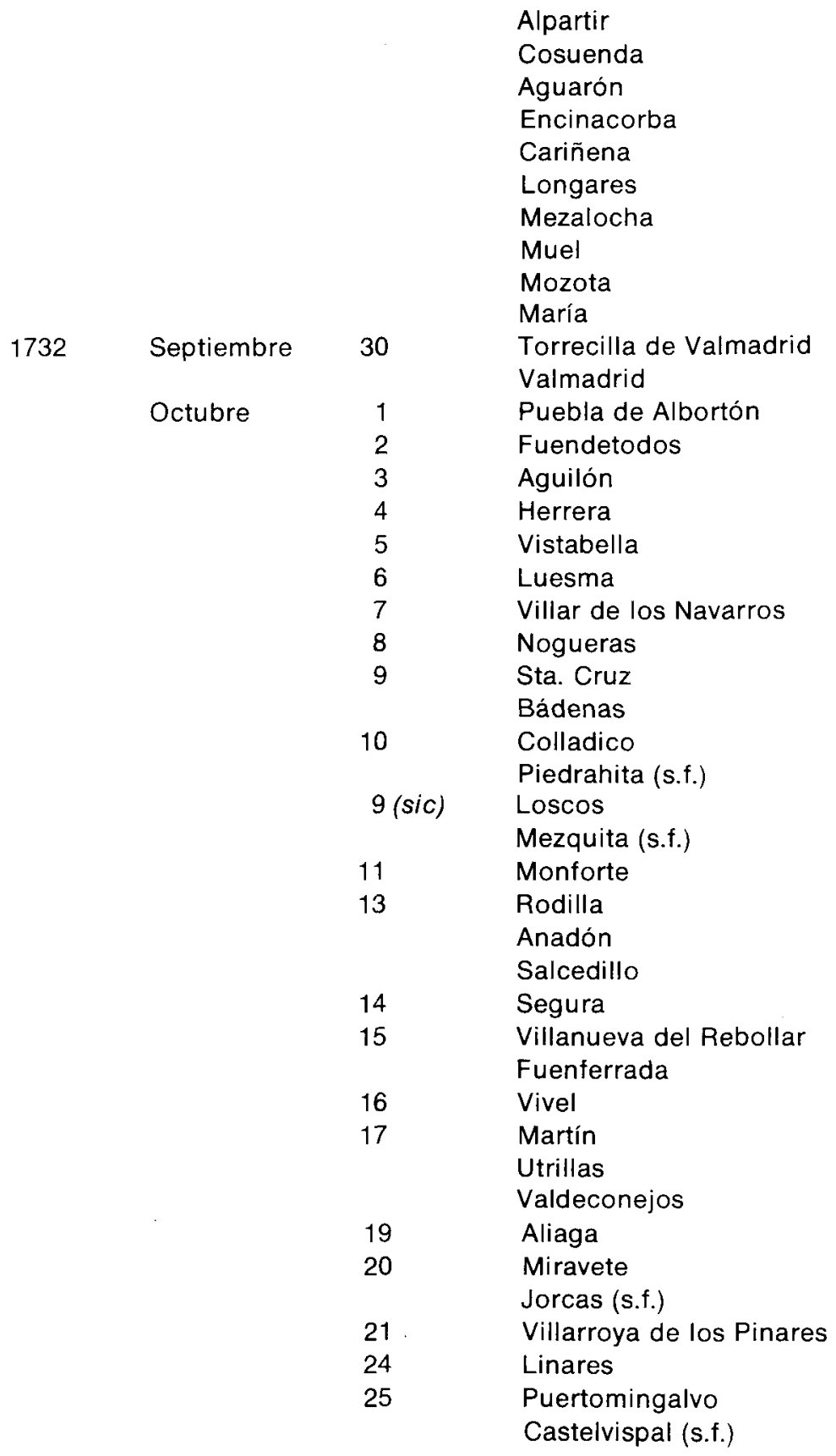




\begin{tabular}{|c|c|c|c|}
\hline & & 28 & Iglesuela \\
\hline & & 30 & Cantavieja \\
\hline & Noviembre & 1 & Fortanete \\
\hline & & & Cañada de Benatanduz (s.f.) \\
\hline & & 2 & Pitarque \\
\hline & & 4 & Montoro \\
\hline & & 5 & Villarluengo \\
\hline & & 6 & Tronchón \\
\hline & & 8 & Mirambel \\
\hline & & & La Cuba (s.f.) \\
\hline & & 10 & Olocau \\
\hline 1732 & Noviembre & 11 & Bordón \\
\hline & & & Luco de Bordón (s.f.) \\
\hline & & & Parras de Castellote (s.f.) \\
\hline & & 12 & Jaganta \\
\hline & & 13 & Castellote \\
\hline & & 14 & Seno \\
\hline & & & Santolea (s.f.) \\
\hline & & 10 & Dos Torres \\
\hline & & 18 & Ladrunan \\
\hline & & 20 & $\begin{array}{l}\text { Molinos } \\
\text { Molan }\end{array}$ \\
\hline & & & Ejulve \\
\hline & & & La Zoma (s.f.) \\
\hline & & 21 & Gargallo \\
\hline & & 22 & $\begin{array}{l}\text { Cañizar } \\
\text { Castel de Cabra }\end{array}$ \\
\hline & & 23 & Palomar \\
\hline & & 25 & Escucha \\
\hline & & 26 & Montalbán \\
\hline & & & Peñarroyas (s.f.) \\
\hline & & 28 & Armillas \\
\hline & & 29 & La Hoz de la Vieja \\
\hline & & 30 & $\begin{array}{l}\text { Josa } \\
\text { Alcaine }\end{array}$ \\
\hline & Diciembre & 1 & Obón \\
\hline & & 3 & Torre las Arcas \\
\hline & & & Estercuel \\
\hline & & 5 & Crivillén \\
\hline & & & La Mata \\
\hline & & 6 & Los olmos \\
\hline & & 7 & Alcorisa \\
\hline & & & Abenfigo (s.f.) \\
\hline
\end{tabular}




\begin{tabular}{|c|c|c|c|}
\hline \multirow[t]{19}{*}{ Año } & Mes & Dia & Parroquia \\
\hline & & 9 & Mas de las Matas \\
\hline & & 10 & Aguaviva \\
\hline & & & Ginebrosa \\
\hline & & & Cañada de Verich (s.f.) \\
\hline & & 11 & Cerollera \\
\hline & & 12 & Monroyo \\
\hline & & & Torre de Arcas (s.f.) \\
\hline & & 13 & Ráfales \\
\hline & & 14 & Fuentespalda \\
\hline & & 15 & Peñarroyas \\
\hline & & & Beceite \\
\hline & & 16 & Valderrobres \\
\hline & & & Torre del Compte \\
\hline & & 17 & La Fresneda \\
\hline & & & Portellada (s.f.) \\
\hline & & 19 & Valjunquera \\
\hline & & 20 & Valdetormo \\
\hline & & & Mas del Labrador \\
\hline \multirow[t]{5}{*}{1732} & Diciembre & 21 & Mazaleón \\
\hline & & 22 & Maella \\
\hline & & 23 & Nonaspe \\
\hline & & 24 & Fabara \\
\hline & & 26 & Alcañiz \\
\hline \multirow[t]{9}{*}{1733} & Enero & 1 & Caspe \\
\hline & & & Chiprana (s.f.) \\
\hline & & 6 & Escatrón \\
\hline & & 7 & Sástago \\
\hline & & & Cinco Olivas (s.f.) \\
\hline & & 8 & La Zaida \\
\hline & & & Quinto (s.f.) \\
\hline & & & Fuentes de Ebro (s.f.) \\
\hline & & 11 & El Burgo \\
\hline \multirow[t]{12}{*}{1733} & Septiembre & 21 & La Muela \\
\hline & & 22 & Epila \\
\hline & & 24 & Salillas \\
\hline & & & Berbedel \\
\hline & & & Lucena \\
\hline & & 25 & Calatorao \\
\hline & & 26 & Alfamén \\
\hline & & 27 & La Almunia \\
\hline & & 30 & Ricla \\
\hline & Octubre & 1 & Arándiga \\
\hline & & 2 & Niguella \\
\hline & & 3 & Mesones \\
\hline
\end{tabular}




\begin{tabular}{|c|c|c|c|}
\hline & & & Brea \\
\hline & & 5 & Illueca \\
\hline & & 6 & $\begin{array}{l}\text { Gotor } \\
\text { Jarque }\end{array}$ \\
\hline & & 8 & Aranda \\
\hline & & & Pomer \\
\hline & & 10 & Trasobares \\
\hline & & 11 & Tierga \\
\hline & & 12 & Tabuenca \\
\hline & & 13 & $\begin{array}{l}\text { Pozuelo } \\
\text { Fuendejalón }\end{array}$ \\
\hline & & 14 & Ainzón \\
\hline & & 15 & $\begin{array}{l}\text { Bureta } \\
\text { Alberite }\end{array}$ \\
\hline & & 16 & Magallón \\
\hline & & 17 & Bisimbre \\
\hline & & & Agón \\
\hline & & & Frescano \\
\hline & & 18 & Mallén \\
\hline & & 19 & Cortes de Navarra \\
\hline & & 20 & Novillas \\
\hline & & 21 & Gallur \\
\hline & & 22 & Boquiñeni \\
\hline & & & Luceni \\
\hline & & & Alcalá de Ebro \\
\hline 1733 & Octubre & 23 & Pedrola \\
\hline & & & Cabañas \\
\hline & & & Figueruelas \\
\hline & & & Grisén \\
\hline & & 24 & $\begin{array}{l}\text { Pleitas } \\
\text { Plasencia }\end{array}$ \\
\hline & & 25 & Lumpiaque \\
\hline & & 24 (sic) & Bardallur \\
\hline & & 26 & Rueda de Jalón \\
\hline & & & Urrea de Jalón \\
\hline & & & Bárboles \\
\hline & & $\begin{array}{l}27 \\
28\end{array}$ & Pinseque \\
\hline & & $\begin{array}{l}28 \\
29\end{array}$ & Monzalbarba \\
\hline & & $\begin{array}{l}29 \\
30\end{array}$ & $\begin{array}{l}\text { Utebo } \\
\text { Sobradiel }\end{array}$ \\
\hline & & 31 & $\begin{array}{l}\text { Sobradiel } \\
\text { Torres de Berrellén }\end{array}$ \\
\hline & & & La Joyosa \\
\hline & & & Marlofa \\
\hline & Noviembre & 1 & Alagón \\
\hline
\end{tabular}


Año

Mes

Día

Parroquia

\begin{tabular}{|c|c|c|c|}
\hline & & 3 & Remolinos \\
\hline & & 4 & Tauste \\
\hline & & 6 & $\begin{array}{l}\text { Ejea de los Caballeros } \\
\text { Rivas (s.f.) }\end{array}$ \\
\hline & & 9 & Erla \\
\hline & & 10 & Luna \\
\hline & & 12 & Valpalmas \\
\hline & & 13 & Pedrosas \\
\hline & & & Sierra de Luna \\
\hline & & 14 & Castejón de Valdejasa \\
\hline & & 15 & Zuera \\
\hline & & 16 & Villanueva de Gállego \\
\hline & & 17 & Peñaflor \\
\hline & & 18 & S. Mateo \\
\hline & & 19 & Leciñena \\
\hline & & 20 & Perdiguera \\
\hline & & 21 & $\begin{array}{l}\text { Villamayor } \\
\text { Pastriz }\end{array}$ \\
\hline & & 22 & Puebla de Alfinden \\
\hline & & & Alfajarín \\
\hline & & 23 & Nuez \\
\hline & & & Villafranca de Ebro \\
\hline & & & $\begin{array}{l}\text { Osera } \\
\text { Aguilar }\end{array}$ \\
\hline & & 24 & Farlete \\
\hline & & 25 & Monegrillo \\
\hline & & 26 & La Almolda \\
\hline & & 28 & Bujaraloz \\
\hline & & 30 & Pina \\
\hline & Diciembre & 2 & Gelsa \\
\hline 1733 & & 3 & Velilla de Ebro \\
\hline & Diclemore & 4 & Alforque \\
\hline & & 5 & $\begin{array}{l}\text { Alborge } \\
\text { Castelnou }\end{array}$ \\
\hline & & & Jatiel \\
\hline & & 7 & Samper de Calanda \\
\hline & & 8 & Puebla de Hijar \\
\hline & & 9 & Híjar \\
\hline & & 11 & Urrea de Gaén \\
\hline & & 13 & Albalate \\
\hline & & 15 & Castelserás \\
\hline & & 16 & $\begin{array}{l}\text { Torrecilla de Alcañiz } \\
\text { Valdealgorfa }\end{array}$ \\
\hline & & 17 & Fórmoles \\
\hline
\end{tabular}




\begin{tabular}{|c|c|c|c|}
\hline Año & Mes & Día & Parroquia \\
\hline & & $\begin{array}{l}18 \\
19 \\
20 \\
21 \\
\\
23 \\
25 \\
27 \\
28 \\
29 \\
30 \\
31\end{array}$ & $\begin{array}{l}\text { Belmonte } \\
\text { Codoñera } \\
\text { Torrevelilla } \\
\text { Foz-Calanda } \\
\text { Calanda } \\
\text { Andorra } \\
\text { Alloza } \\
\text { Ariño } \\
\text { Oliete } \\
\text { Alacón } \\
\text { Muniesa } \\
\text { Plou }\end{array}$ \\
\hline 1734 & Enero & $\begin{array}{r}1 \\
2 \\
3 \\
5 \\
6 \\
7 \\
8 \\
9 \\
10 \\
\\
11 \\
12 \\
13 \\
14 \\
15 \\
18\end{array}$ & $\begin{array}{l}\text { Cortes de Aragón } \\
\text { Maicas } \\
\text { Huesa } \\
\text { Blesa } \\
\text { Moneva } \\
\text { Moyuela } \\
\text { Plenas } \\
\text { Azuara } \\
\text { Samper del Salz } \\
\text { Letux } \\
\text { Lagata } \\
\text { Lécera } \\
\text { Belchite } \\
\text { Almonacid de la Cuba } \\
\text { Azaila } \\
\text { Vinaceite } \\
\text { Codo } \\
\text { Mediana }\end{array}$ \\
\hline
\end{tabular}




\section{NOTAS}

(1) Vid. el mapa y el itinerario que adjuntamos.

(2) Creemos que inició esta visita general a finales del mes de septiembre porque en el folio 8 hay una mención del mes y día que se estuvo en la parroquia de Cerveruela, concretamente el 1 de octubre; y en el folio 17, en Cutanda, cita sólo el día, era el día 12.

(3) La fiabilidad de esta fuente va avalada por el estudio realizado en nuestra tesis doctoral sobre la visita del arzobispo Añoa «Iglesia y sociedad zaragozana a mediados del s. XVIII. Aspectos geográficos, demográficos, sociológicos, eclesiásticos y fiscales de la diócesis de Zaragoza a través de una visita pastoral del prelado Francisco Añoa (1745-1749)", leida en la Facultad de Filosofía y Letras de la Universidad de Zaragoza durante el curso académico 1980-81. Fue dirigida por el doctor don Angel Canellas López; o bien cotejando los mandatos de visita que se conservan en las parroquias en los que consta la fecha en que se visitaron.

(4) Se conserva, en el Archivo Diocesano de Zaragoza de las visitas efectuadas durante e gobierno de este prelado, el libro de los derechos de la visita realizada por don Juan del Cotero los años 1736-37, que fue objeto de una comunicación nuestra titulada uUn itinerario de visita pastoral a la diócesis de Zaragoza basado en el libro de los derechos de visita: años 1736-37" (en prensa), presentada al Simposio Nacional de ciudades episcopales, celebrado en Tarazona los dias 9 al 11 de diciembre de 1982.

(5) Hemos cotejado los mandatos de visita de las parroquias de Castejón de Valdejasa (cfr. Archivo Parroquial de Castejón de Valdejasa. «Libro de bautizados, confirmados, matrimonios y difuntos y cumplimiento pascual». Años 1676-1733. Tomo 3.9) y Pedrola (cfr. Archivo Parroquial de Pedrola. «Libro de registro de Difuntos. Años 1733-1775. ff. 5'-7' $)$ ).

(6) A mediados del siglo XVIII el Arzobispado de Zaragoza estaba dividido eclesiásticamente en tres Arciprestazgos: Arciprestazgo de Alcañiz, de Zaragoza y de Daroca (cfr. nuestra tesis doctoral en la que damos la relación de las parroquias que pertenecían a cada uno de ellos).

(7) La fuente documental no cita a Almonacid de la Sierra y a Botorrita como parroquias visitadas.

(8) En la fuente documental no consta Roden como parroquia visitada.

(9) Así se puede comprobar en el estudio de las visitas llevadas a cabo a la diócesis de Zaragoza en los años 1736-37 y 1745 a 1749 (cfr. nuestra tesis doctoral y nuestro artículo citado en la nota 4). Si bien hay que destacar que los derechos de pila y dieta pueden hallarse mencionados en dos de estos libros.

(10) Constituciones synodales de el Arçobispado de Zaragoça. Hechas y ordenadas por... D. Antonio lbáñez de la Riva Herrera, Arçobispo... en el sinodo... del año 1697, Zaragoza, 1698 , L. 4 T. 3 C. I, p. 536.

(11) Ibidem, L. 4 T. 3 C. I, p. 537.

(12) lbidem, L. 2 T. 10 C. I, p. 324. La letra Y que encontramos con frecuencia precediendo al nombre indica los que han sido enterrados en la iglesia.

(13) Ibidem, L. 4 T. 3 C. I, p. 536. 